\author{
Claus C. Schnorrenberger
}

\section{Chinesische Medizin und Akupunktur} im Westen

Chinesische Medizin und Akupunktur werden von orthodoxen westlichen Medizinern als von nicht besonders hoher wissenschaftlicher Qualität eingeschätzt und herablassend belächelt; man hält sie für wenig mehr als gute Placebos. Deshalb fordern westliche Wissenschaftler und Gesundheitspolitiker, dass beide Methoden rigorosen, auf Evidenz zielenden Tests unterworfen werden, die möglichst alle bekannten medizinischen Diagnosen erfassen sollten, was auf lange Sicht mühsam, zeitaufwendig und kostspielig werden dürfte. Andererseits erfreuen sich die beiden Heilmethoden bei westlichen (und fernöstlichen) Patienten grosser Beliebtheit. Verglichen mit modernen westlichen Behandlungsmethoden zeigen sie, im Gegensatz zu westlichen pharmazeutischen Präparaten, nur geringe Nebenwirkungen, und sie können zahlreiche Krankheiten heilen, gegen die die westliche Medizin machtlos ist. Im Jahr 2009 brachte ein Referendum in der Schweiz das Ergebnis, dass sich 67\% der Abstimmenden komplementäre medizinische Behandlungen wünschten, von denen die chinesische Medizin ein Teilgebiet ist [3]. Dennoch behaupten manche Politiker, das allgemeine Publikum sei zu unwissend, um die einschlägigen wissenschaftlichen Probleme richtig zu beurteilen, obwohl das gleiche Stimmvolk nicht zu dumm war, für eben die Politiker zu votieren, die jetzt seinen Wunsch ignorieren möchten.

\title{
Wissenschaftstheoretische Bewertung der chinesischen Medizin und Akupunktur - Teil 1
}

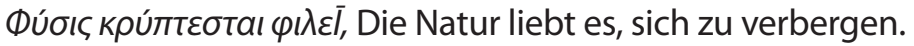 \\ (Heraklit, 544-483 v.Chr. [1])
}

Es ist vermutlich ganz allgemein wahr, dass sich die fruchtbarsten Entwicklungen in der Geschichte des menschlichen Denkens häufig an solchen Stellen ereignen, wo zwei verschiedene Richtungen des Denkens zusammentreffen. Diese Richtungen können ihre Wurzeln in ganz verschiedenen Gebieten der menschlichen Kultur haben, in verschiedenen Epochen, verschiedener kultureller Umgebung oder verschiedenen religiösen Traditionen; aber wenn sie wirklich zusammentreffen, das heisst, wenn sie mindestens so sehr miteinander in Beziehung stehen, dass eine echte Wechselwirkung erfolgen kann, dann darf man hoffen, dass neue und interessante Entwicklungen folgen. (Werner Heisenberg [2])

Vor 9 Jahren wurde in Deutschland eine Akupunkturstudie durchgeführt, die sogenannten GERAC- und ART-Tests [4]. Sie wurden als die grössten jemals durchgeführten Untersuchungen über das Fachgebiet bejubelt. Etwa 100000 Patienten, die an den häufig vorkommenden Krankheiten Rückenschmerzen und Osteoarthritis des Kniegelenks litten, wurden mit echter Akupunktur, einer Placebo-Akupunktur und, zum Vergleich, mit Methoden der Schulmedizin behandelt. Eine sogenannte «echte» und eine «Placebo»-Akupunktur-Gruppe von Patienten wurden gebildet, und eine dritte Gruppe aus Probanden, die an denselben beiden Krankheiten litten, wurde aufgestellt und mit üblicher Schulmedizin behandelt. Die Ergebnisse der drei Gruppen wurden verglichen. Erstaunlicherweise zeigte die Gruppe unter «echter» Akupunktur eine Erfolgsrate von etwa $47,6 \%$, die Placebo-Gruppe erreichte eine Quote von 44,2\%, während die schulmedizinisch behandelte
Gruppe nur einen Erfolg von 27,4\% verbuchen konnte [5]. Somit hatte die Studie ein verwirrendes Resultat. Das führte einige Beobachter $\mathrm{zu}$ dem Schluss, dass nun jedermann erlaubt sein müsse, Nadeln zwecks Krankheitsbehandlung in den menschlichen Körper zu stechen, egal wohin, und es führte zur Vermutung, dass die überlieferten Lehren und Regeln der Akupunktur überflüssig seien [6-8].

Das Verwirrspiel hat $\mathrm{zu}$ widersprüchlichen Debatten unter Fachleuten und im Publikum geführt. Ein Ergebnis war, dass deutsche Krankenkassen nun ca. 50 Euro für jede derartige Farce in Arztpraxen zu berappen hatten. Weil solches in Deutschland täglich tausendfach geschieht, sind die Behandlungsergebnisse kläglich, und die winzige Schar kenntnisreicher deutscher Akupunkteure wird immer kleiner. Kurzum: Quacksalberei mit Akupunktur ist angesagt, während die GERAC-Studie rund stolze 10 Millionen Euro verschlungen haben dürfte. Diese

\section{KARGER}

Fax +497614520714 Information@Karger.de www.karger.com
(๑) 2011 S. Karger GmbH, Freiburg

Accessible online at: www.karger.com/szg
Prof. h.c. (China Medical University, Taichung, Taiwan) Dr. med. Claus C.

Schnorrenberger, M.D.

Lifu International College of Chinese Medicine (LICCM)

Karl Jaspers-Allee 8, 4052 Basel, Schweiz

Tel. +41 61 37330-78, Fax -79

lifu@gmx.ch 
Summe wurde aus den eingezahlten Vermögen der krankenversicherten Bürger bezahlt, ohne dass letztere vorher über die Verschwendung ihrer Gelder informiert worden wären.

Der vorliegende Artikel versucht, eine Erklärung für die Hintergründe dieses Missstands zu geben (der übrigens in anderen europäischen Ländern ähnlich ist), um weiteren Fehlinterpretationen und nutzlosen Geldausgaben Einhalt zu gebieten.

Meine Argumentation beginnt mit den urkundlichen Anfängen der chinesischen Medizin und Akupunktur und zugleich mit Dokumenten vom Beginn der wissenschaftlichen Beweisführung im Abendland, um Licht in die offensichtlich verfahrene Situation zu bringen. Der Autor hofft, zeigen zu können, dass das originale Konzept der chinesischen Heilkunde und Akupunktur und der Anfang wissenschaftlicher Logik im Abendland Gemeinsamkeiten haben, die genügend Substanz enthalten, um zu einer notwendigen logischen Einsicht zu gelangen.

\section{Krankengeschichte 1}

Im Jahr 1988 hatte ich einen Patienten zu behandeln, der nach einer Non-ANon-B-Hepatitis an einer schweren Leberzirrhose litt. Er war depressiv und verängstigt, als er zur ersten Konsultation kam, nachdem ihm ein berühmter Professor der Universitätsklinik prophezeit hatte, er müsse binnen weniger Wochen sterben, weil für ihn keinerlei Therapie möglich sei ausser eventuell einer Lebertransplantation; doch es war kein Organspender in Sicht.

Ich sah mir den Mann an, der um die Mitte 40 war. Die Skleren seiner Augen waren dunkelgelb, sein Gesicht war ebenfalls gelb, seine Lippen und seine Zunge zeigten eine massive Gelbfärbung. Seine Wangenknochen ragten aus dem abgemagerten Gesicht. Er sagte, er fühle sich hunds- miserabel; er habe $20 \mathrm{~kg}$ Gewicht verloren, sein Appetit sei völlig weg. Er leide ständig unter Kopfweh, Schwindel und Magenschmerzen, nachts könne er nicht schlafen. Sein Hausarzt schickte ihn zu mir, weil der wusste, dass ich mehrere Hepatitis-Patienten erfolgreich mit Akupunktur behandelt hatte. Der Patient hatte seine Laborwerte mitgebracht: Das Bilirubin betrug 23,7; GPT und GOT lagen um 600; das Gamma-GT war 230.

Ich erklärte ihm als Erstes, ich könne nicht verstehen, warum ihm die Klinikärzte den baldigen Tod vorausgesagt hatten, und schlug vor, ihm eine Reihe von Akupunkturen und chinesische Kräuterrezepturen $\mathrm{zu}$ geben, um seinen Zustand zu bessern.

«Meinen sie, ich hätte noch eine Chance?», fragte er ängstlich.

«Durchaus!», war meine Antwort. «Ihr schlauer Professor könnte früher sterben als Sie!» Der Kranke schöpfte etwas Hoffnung.

Ich besah mir seine Zunge genauer, fühlte seinen Puls, bat ihn, sich zu entkleiden, und untersuchte ihn von Kopf bis Fuss. Dabei betastete ich die druckschmerzhaften Stellen an seinem Körper; es waren die Akupunktur-Strukturen, in die ich meine Nadeln $\mathrm{zu}$ stechen hatte: Jimen 期門 (Leber 14), Riyue 日月 (Gallenblase 24), Zhongwan 中脘 (Renmai 12), Sanyinjiao 三陰交 (Milz 6), Ganyan 肝炎 (zusätzlicher Punkt am Unterschenkel), Taichong 太冲 (Leber 3), Yanglingquan 陽陵泉 (Gallenblase 34), Neiguan 內關 (Perikard 6), Baihui 百會(Dumai 20) an seiner Vorderseite; $X i n s h u$ 心腧 (Blase 15), Shenshu 腎腧 (Blase 23), Pishu 脾腧 (Blase 20), Weizhong 委中 (Blase 40) am Rücken. Ich schrieb seine Bian-Zheng-Analyse in sein Krankenblatt: "Nässe und Hitze in Leber und Gallenblase» (Gan Dan Shi Re 肝膽濕熱) und begann mit der Akupunktur. Danach fühlte er sich etwas besser, war aber sehr müde.
In den folgenden Tagen kam er zweimal wöchentlich. Nach 2 Wochen war das Bilirubin von 23,7 auf 12 gesunken, GOT und GPT waren auf 200 gefallen, das Gamma-GT betrug nur noch 80, und er war weniger gelb. Einen Monat später lag das Bilirubin bei 7,5, GOT und GPT lagen bei 69 , das Gamma-GT war 45. Sein Appetit war wieder da, er schlief besser, und der Schwindel war ganz verschwunden. Ich schrieb ihm zusätzlich eine Rezeptur aus chinesischen Heilkräutern. Er wunderte sich, dass er noch am Leben war; seine Hoffnung wuchs von Tag zu Tag. Er hatte zugenommen und fühlte sich viel besser. 6 Monate später konnte er in seine Berufstätigkeit zurückkehren. Die Krankenkasse ersetzte ihm keinen Pfennig seiner Behandlungskosten, weil sie behauptete, es habe sich bei meiner Akupunktur und den Kräuterrezepten um eine völlig «unwissenschaftliche» Behandlungsmethode gehandelt, und seine Heilung sei deshalb nur ein «unspezifischer» Effekt gewesen. Er verklagte die Kasse und gewann schliesslich seinen Prozess in der zweiten Instanz, nachdem er dem Richter erklärt hatte, er sei lieber «unwissenschaftlich lebendig als wissenschaftlich tot». Der Richter besass so viel Einsicht, ihm das zu glauben.

Ein Jahr danach erlitt er einen leichten Rückfall, den ich ebenfalls erfolgreich behandelte. Er war glücklich und heiratete seine langjährige Lebensgefährtin. $\mathrm{Zu}$ Weihnachten schrieb er mir eine Karte, auf der er betonte, die wichtigste Hilfe für ihn sei meine Mitteilung gewesen, er habe eine Chance, weiterzuleben, und dass er mir vertraut hätte. Er lebte weitere 15 Jahre und kam gelegentlich zur Auffrischungsbehandlung in die Praxis. Einer seiner pessimistischen Professoren war inzwischen verstorben, sodass er annahm, ich hätte auch ein prophetisches Talent.

Wie konnte das alles passieren, und wie ist es zu erklären? Ich werde versuchen, es auf den folgenden Seiten zu 
analysieren und wissenschaftlich zu untermauern.

\section{Die Wurzeln der chinesischen Akupunktur}

Im 1. Kapitel (Abb. 1) des Buches Huangdi Neijing Lingshu 黄帝內經霝 樞 (Lingshujing 霝樞經), betitelt Die Neun Nadeln und die Zwölf Yuan (九 針十二原 Jiu Zhen Shi Er Yuan), betont der kaiserliche Arzt Qi Bo 歧伯 in einem Dialog mit dem Gelben Kaiser Huang-Di (Abb. 2), dass Shen 神 das wichtigste Phänomen sei, das vom Arzt verstanden und befolgt werden müsse, wenn er Akupunktur praktiziert [9].

Das Schriftzeichen Shen 神 ist zusammengesetzt aus 示 shi und 申 shen. Shi 示 besteht aus 二, einer alten Form für «oben» oder «Himmel» (die in modernem Chinesisch 上 geschrieben wird), und aus III chuan, was heisst: «Fluss», etwas, das «fliesst». Demnach ist Shi 示 nach
Dr. Leon Wieger das, was vom Himmel herabfliesst, nämlich die Sonne, der Mond, die Sterne. Mit anderen Worten: Shi 示 symbolisiert die Ordnung im Universum, den Kosmos. Der zweite Teil des Schriftzeichens Shen 神 ist der phonetische Bestandteil 申 shen, der «unterrichten» oder «Information geben» bedeutet. Demzufolge heisst Shen 神 «Aufschluss geben über das, was vom Himmel herunterfliesst», nämlich Sonne, Mond und Sterne, mit anderen Worten «Informieren über die Ordnung des Universums» [10], im abendländischen Sinne frei übersetzt: «Aufklärung geben über die Natur». Die heute übliche Wiedergabe von Shen 神 mit «Geist» oder «Seele» ist eine sehr verkürzte Beschreibung, die die originäre $\mathrm{Be}$ deutung nur partiell erfasst.

Wenn wir Dr. Leon Wiegers Interpretation von Shen 神 folgen [10], finden wir darin ein Verständnis des Ganzen, von dem das menschliche Wesen und seine Individualität dedu- ziert werden müssen, bevor Akupunktur professionell ausgeführt werden kann. Shen 神 (Abb. 3) ist die Ordnung des Universums, der Kosmos, in dem die Menschheit und der einzelne Mensch integrierte Teile sind. Darin liegen die Wurzeln der chinesischen Akupunktur, und daher stammen ihre Grundannahmen und Vorschriften [11].

Die logische Methode der Deduktion ist in der Tradition chinesischen Denkens verankert. Sie wird z.B. von Lao $\mathrm{Zi}$ 老子im Kapitel 42 四十二 章 seines Dao De Jing 道德經 verwendet. Dao heisst nicht nur «Weg», sondern auch Wahrheit, Doktrin, Prinzip, Vernunft [12]. Lao Zis Vers [13] (Abb. 4) kann demnach folgendermassen übersetzt werden:

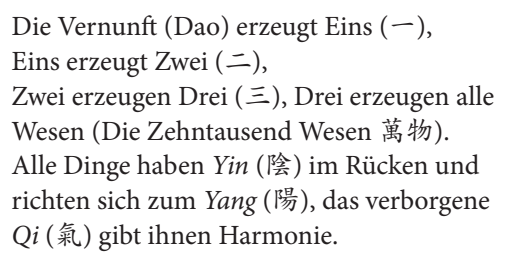

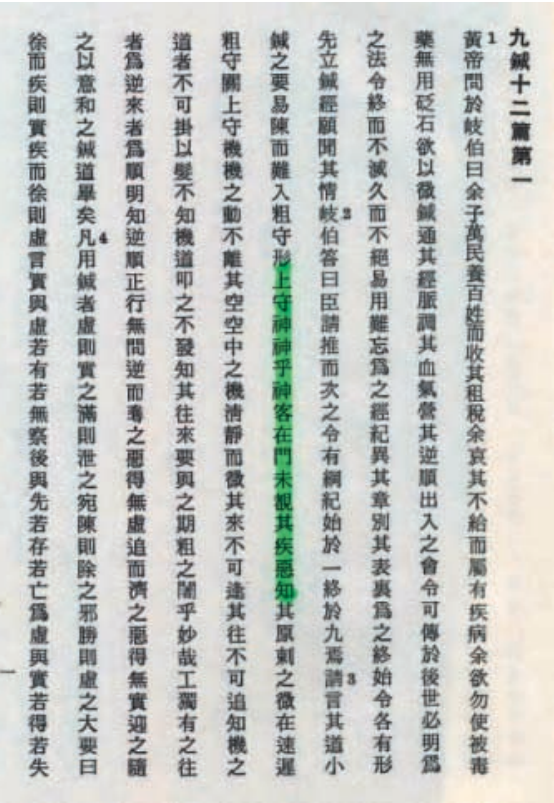

Abb. 1. Erste Seite von Kapitel 1 des Lingshujing 霝樞經: Die Neun Nadeln und die Zwölf Yuan (Jiu Zhen Shi Er Yuan 九 針十二原). Hier wird die Bedeutung des Shen 神 für die Praxis der Akupunktur erklärt [9].

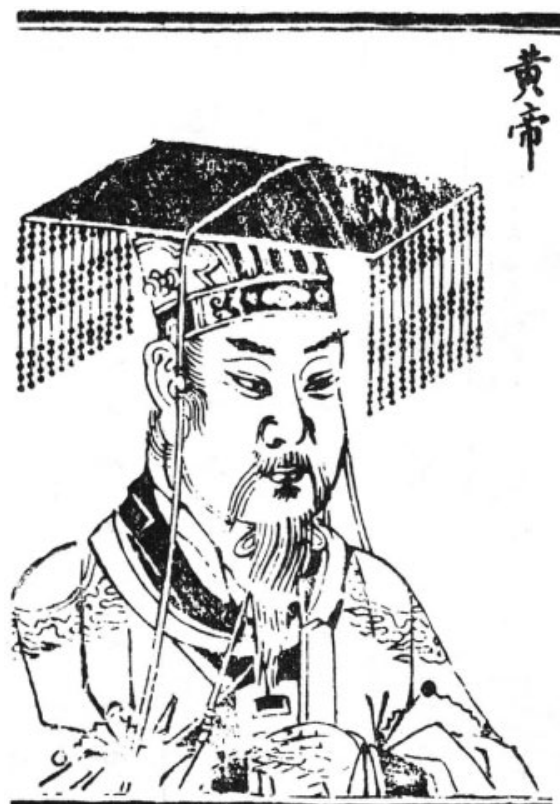

Abb. 2. Kaiser Huang-Di lebte nach der Legende im 27. Jahrhundert v.Chr.

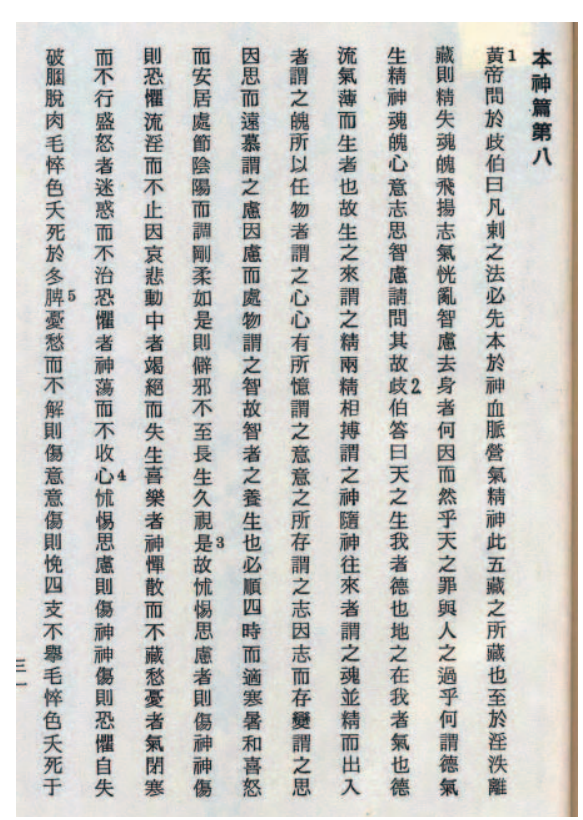

Abb. 3. Erste Seite von Kapitel 8 des Lingshujing: Der Ursprung Shen (Ben Shen 本神). Himmel (Tian 天), Tugend ( De 德), Erde ( $i$ 地), Q $i$ (氣), Jing (精) und weitere Untergruppen wie Hun 魂, $P o$ 魄, Xin 心, $Z h i$ 志 und $S i$ 思 werden von Shen 神 deduziert [11]. 


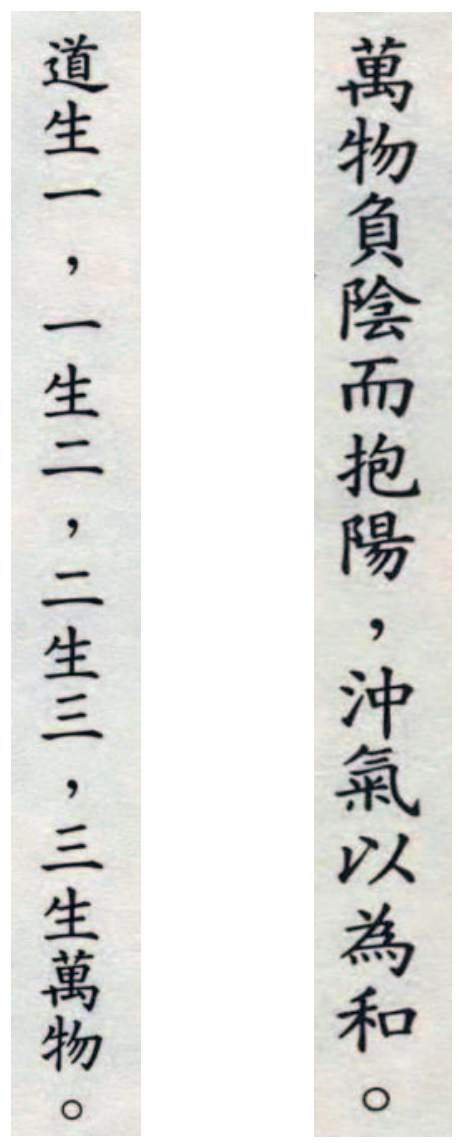

Abb. 4. Lao Zis Vers im Kapitel 42 四十二章 seines Dao De Jing 道德經 [13].

Hier sind Eins, Zwei, Drei und schliesslich «alle Dinge des Universums» Ergebnisse der Vernunft, der Wahrheit, des ersten Prinzips, des $D A O$ 道.

Die chinesischen Ziffern Eins, Zwei, Drei repräsentieren zusätzlich zu ihrer Zählfunktion umfassende Phänomene. Eins, $-Y i$, steht für das Ganze der Welt, für die Einheit des Kosmos; Zwei, 二 Er, vertritt Yang und Yin, Himmel und Erde; Drei, 三San, symbolisiert die Menschheit. Das alte chinesische Ordnungsemblem Tian-Ren-Di 天人地 bedeutet den Menschen, die Menschheit, die zwischen Himmel und Erde steht.

Diese Auffassung ist im zitierten Vers des Lao Zi und in der Philosophie des Huangdi Neijing Lingshu enthalten. Dem entsprechend ist die Akupunktur ein medizinisches Verfahren, das auf der Logik der Deduktion beruht (nahezu alle westlichen Autoren der chinesischen Medizin und Akupunktur haben diese einfache, aber grundlegende Tatsache übersehen). $\mathrm{Zu}$ sämtlichen diagnostischen und therapeutischen Methoden der chinesischen Medizin gelangt der chinesische Arzt über die Deduktion aus Yin-Yang. Die Gefässe des menschlichen Organismus, die 12 Jingmai 經脈 (Blutgefässe), sind ebenso wie die Zang-Fu 藏腑, d.h. die inneren Organe, entsprechend der Yin-YangVerteilung angeordnet. Auch die sechs klimatischen Störungen Liu Yin 六淫, die Acht Leitprinzipien $B a$ Gang 八網 und das Bian-Zheng 辨證, das der Schlüssel zur chinesischen Krankheitserkennung ist, ohne den in der chinesischen Heilkunde gar nichts läuft, beruhen auf Yin-Yang, wobei Zheng 證 Evidenz bedeutet, was im Abschnitt über Evidence-Based Medicine (EBM) weiter diskutiert wird.

Nebenbei sei angemerkt, dass auch die Schriftzeichen der chinesischen Schrift deduktiv konstruiert sind. Sie zeigen ihren Ursprung, das Radikal oder Wurzelzeichen (Bushou 部首), wohingegen die Wörter westlicher Sprachen aus 26 Buchstaben induktiv zusammengesetzt sind. Unter anderem deshalb sind sie manchmal mehrdeutig und können zu Trugschlüssen in der geschriebenen Sprache führen, worauf Aristoteles in seinen Sophistischen Widerlegungen (Sophisticōn

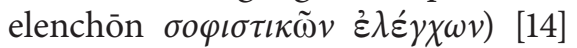
hingewiesen hat. Derartiges passiert mit geschriebenen chinesischen Zeichen selten oder gar nicht.

\section{Die Anfänge wissenschaftlicher Logik im Westen}

Deduktion ist, zusätzlich zur Induktion, nicht nur ein wichtiges logisches Verfahren in der chinesischen Tradition, sondern auch ein Prüfstein abendländischer Logik. In seiner Vorlesung über Natur ( $\Phi \Upsilon \Sigma I K H \Sigma$ $A K P O A \Sigma E \Omega \Sigma$ ) stellt Aristoteles fest, dass ein verlässlicher Beweis nur durch Deduktion (syllogismos $\sigma v \lambda \lambda o \gamma ı \sigma \mu o ́ s)$ aus einer Substanz

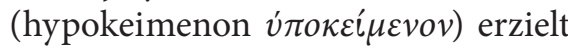
werden könne. Er bezeichnet die Teile bzw. die Attribute, die aus einer Substanz deduziert sind, als Akzidenzien (symbebēkos $\sigma v \mu \beta \varepsilon \beta \eta \kappa o ́ \varsigma)$ [15] und erklärt, dass Akzidenzien kein eigenes Sein besitzen. In seiner Metaphysik behauptet er, dass durch blosse Induktion mittels Akzidenzien kein gültiger wissenschaftlicher Beweis zu erzielen sei [16]. Letzteres wurde später von anderen westlichen Philosophen wie Francis Bacon und John Stuart Mill bezweifelt. Um den wissenschaftstheoretischen Grund freizulegen, auf dem die chinesische Medizin beruht, ist es indessen wichtig, Aristoteles' Position hier zu betonen, weil beide Ansatzpunkte ähnlich $\mathrm{zu}$ sein scheinen.

Andererseits verlässt sich die moderne empirische Forschung vor allem auf induktive Folgerungen, etwas, das $\mathrm{zu}$ neuen Erfindungen beitragen kann, die letztendlich aber nicht absolut sicher sind. Das zeigen die Nebenwirkungen moderner pharmazeutischer Präparate ebenso deutlich wie allfällige nukleare Katastrophen. Jedenfalls ist das deduktive Vorgehen die hauptsächliche methodische Quelle der chinesischen Heilkunde im Hinblick auf die Morphologie, Physiologie, Pathologie, Diagnostik, Akupunktur, Moxibustion und Rezeptur von Heilkräutern, was noch eingehender behandelt werden soll. (Ich behaupte, dass Deduktion und Induktion gemeinsam angewandt werden müssen, wobei der Deduktion die Schlüsselrolle zukommt, wenn es darum geht, logisch gerechtfertigte und wissenschaftlich makellose Resultate $\mathrm{zu}$ erzielen, während die Induktion nur technische Einzelerkenntnisse liefern kann.)

Wissenschaft (epistēmē è wurde erstmals im griechischen Altertum artikuliert. Nachdem die Vorsokratiker Alkmaion, Kratylos, Demokrit und die Pythagoräer den 
Begriff geschaffen hatten [17], verwendet Aristoteles das Wort «epistēmē» wiederholt in seiner genannten Vorlesung, ferner in der Zweiten Analytik (ANA $\Upsilon T I K \Omega N ~ \Upsilon \Sigma T E P \Omega N)$ und in den Topica (TOПIK $\Omega N$ ) [18-20].

Die Bedeutung von «episteme» ist seither gültig geblieben. Ich sage dies trotz Poppers Anmerkung, dass sich «das alte wissenschaftliche Ideal der epistēmē - des absolut sicher demonstrierbaren Wissens - als Idol (d.h. als Götzenbild) erwiesen hat» [21].

Es ist erstaunlich, dass Aristoteles in seiner Vorlesung über die Natur (Abb. 5) ein ähnliches Vorgehen empfiehlt, wie es von Qi Bo im 1. Kapitel des Ling-Shu-Jing bezüglich der Akupunktur gefordert wird:

In allen Wissenschaften, die sich mit Grundsätzen, Ursachen oder Grundelementen befassen, ist es bedeutsam, dass eben diese Grundsätze bekannt sind. Denn wir sind von der Kenntnis einer Sache erst dann überzeugt, wenn wir mit ihren ersten Ursachen und Prinzipien vertraut und zu ihren Grundelementen vorgedrungen sind. Darum ist klar, dass auch bei der Wissenschaft von der Natur zunächst Grundsätze zu bestimmen sind. So ergibt sich der Weg der Untersuchung von dem uns unmittelbar Erkennbaren und Klaren zu dem nach seiner Natur Klareren und von innen her Erkennbaren. Denn das, was unserer Kenntnis direkt zugänglich ist, und das, was nur von innen her (nach seiner Natur) verstehbar ist, ist nicht dasselbe. Deshalb müssen wir folgendermassen vorgehen: Von dem uns zunächst Erkennbaren müssen wir hin zu dem nach seiner Natur und von innen her Klareren gehen, weil das nur seiner Natur Gemässe unserem Verständnis leichter zugänglich ist.

Nun sind die uns unmittelbar verständlichen Dinge aber eher materiell und vereinzelt als abstrakt und grundsätzlich, während ihre Grundelemente und Prinzipien erst später bekannt werden, nämlich erst dann, wenn wir die vereinzelten materiellen Dinge analysiert haben. Deswegen muss der Weg von den Ganzheiten zu den Einzelheiten führen, die von dieser Ganzheit umfasst werden. Denn nach unserer Sinneswahrnehmung ist stets das Ganze leichter erkennbar; und indem ich das gesamte Konkrete, Materielle ein Ganzes nenne, drücke ich damit aus, dass es einen gesamten Komplex umfasst, eine Vielheit von zusammenhängenden Einzelelementen, Faktoren und Eigenschaften, eben seine Teile [22].

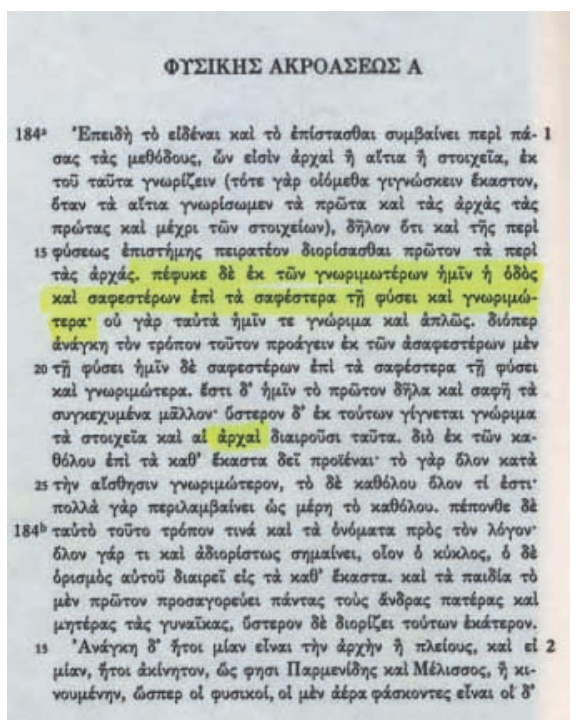

Abb. 5. Erste Seite der Vorlesung über die Natur (Physik) des Aristoteles, $\Phi Y \Sigma I K H \Sigma$ $A K P O A \Sigma E \Omega \Sigma$, Buch I, Kapitel 1 [20].

Aristoteles' Feststellung wurde als Spruch «Das Ganze ist mehr als die Summe seiner Teile» popularisiert.

Von einem massgeblichen abendländischen Philosophen ausgedrückt, beschreibt dieser Text das gleiche Problem wie die Aussage des Qi Bo im 1. Kapitel des Ling-Shu-Jing, dass Akupunktur auf dem Shen 神 beruhen muss:

- Qi Bo fordert, dass der Akupunkteur zunächst die Ordnung des Kosmos, das Shen 神, verstehen muss, das eine umfassende Ganzheit ist, von der alle weiteren Teile der chinesischen Medizin abgeleitet werden.

- Aristoteles argumentiert, dass wir beim Studium der Natur zu einem Ganzen zurückgehen müssen, zum

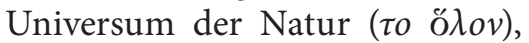
und dass wir seine Teile daraus deduzieren sollen [23].

Somit ist Shen 神 im Wesentlichen identisch mit der Natur (Physis $\varphi v ́ \sigma \iota \varsigma$ ) des Aristoteles, wenn er über das Ganze ( $\tau o$ ő $\lambda o v$ ) der Natur spricht. Beide Feststellungen bilden eine authentische Grundlage und einen unbezweifelbaren Hintergrund, ein Prinzip und Axiom für die daraus folgenden Konsequenzen.

\section{Krankengeschichte 2}

Eine Frau vom Lande kam 1976 in Begleitung ihrer dreijährigen Tochter in die Praxis. Sie sah bleich und aufgedunsen aus, verbreitete einen durchdringenden Uringestank und stöhnte: «Nach Auskunft der Universitätsklinik muss ich in Kürze sterben, weil ich dicke Steine in beiden Nieren habe. Man kann mir nicht helfen, weil sie nicht die eine Niere entfernen können, solange die andere auch nicht richtig arbeitet.» Ich sah und roch, dass sie an einer schweren Urämie litt. Sie zeigte mir ihre Röntgenbilder, die man ihr zu meinem Erstaunen ausgehändigt hatte mit der Information, sie möge ihr Glück anderswo versuchen. Die rechte Niere war total von einem grossen Ausgussstein blockiert, die linke war halb mit Konkrementen angefüllt. Sie sagte: «Ich weiss, Sie können mir helfen, Herr Doktor. Ich habe die ganze Zeit zum Herrgott gebetet, dass er mich noch eine Weile leben lässt, nicht meinetwegen, sondern wegen meiner Kleinen, die mich brauchen. In der Zeitung habe ich einen Artikel über Sie und Ihre Akupunktur gelesen.»

Ich zögerte, sie zu behandeln, weil ich einem derartigen Fall noch nie begegnet war. Ich sagte ihr deshalb, ich würde mich gern noch mit einem befreundeten Kollegen beraten, einem Urologen, bevor ich dann vielleicht mit einer Behandlung beginnen könnte. Das kleine Mädchen hatte angefangen zu weinen; es umklammerte ängstlich die Knie der Mutter. Ich fühlte den Puls der Frau, besah ihre Zunge und schrieb das Bian-Zheng in ihr Krankenblatt: "Leere des Yin der Niere (Shen Yin Xu 腎陰虛)».

«Verlieren Sie bitte keine Zeit, Herr Doktor», beharrte die Patientin. «Fangen Sie mit der Behandlung an; ich weiss, Sie können es schaffen!»

Ich bat sie, die Kleider abzulegen und sich auf eine Liege zu legen. Ihre Unterschenkel und Füsse waren stark angeschwollen. Ihr Unterbauch wölbte sich stark vor; er war ganz mit 
Flüssigkeit angefüllt, einem Aszites, der von ihren funktionsuntüchtigen Nieren kam. Ich dachte, ich wollte ihr wenigstens zeigen, dass Akupunktur in ihrem Fall nicht das Richtige war. Ich wählte die Einstichstellen nach dem Ling-Shu-Jing und behandelte zuerst ihre Vorderseite, dann den Rücken. Als ich fertig war, kam ihr Mann; er holte sie ab und fuhr sie mit dem Kind nach Hause. Das Ehepaar lebte mit seinen vier Kindern etwa $30 \mathrm{~km}$ ausserhalb der Stadt in einem Dorf. Er rief mich am folgenden Morgen an und berichtete, seine Frau habe während der Nacht weniger Schmerzen gehabt, sie sei aber äusserst schwach und könne nicht aufstehen. Deshalb fuhr ich abends nach der Sprechstunde hinaus und gab ihr eine zweite Behandlung. In den folgenden Tagen fuhr ich jeden Tag zu ihr. Ihr Appetit kam zurück, sie schlief besser, sie konnte mehr und leichter Urin lassen ohne Schmerzen oder beigemengtes Blut. Ihr unangenehmer Uringeruch verschwand, und nach 2 Wochen entdeckte ich, als sie sich umdrehte, auf ihrem Betttuch ein kleines dunkelgrünes Bröckchen. Ich fragte, ob sie im Bett etwas gegessen hätte.

«Diese kleinen Dinger kommen jetzt immer wieder heraus», sagte sie, «oft, wenn ich Wasser lasse, manchmal auch im Schlaf.»

Ich untersuchte das Steinchen und sagte: «Nehmen Sie ein grosses Einmachglas und sammeln Sie alle abgehenden Steine darin!»

Nach 4 Wochen war das Glas halb mit schwarzgrünen Nierensteinen angefüllt, jeder etwa so gross wie ein Reiskorn. War es möglich, dass meine Akupunktur die Chemie ihres Blutes so verändert hatte, dass ihr Körper die Nierensteine von selbst auflösen konnte? Daheim wiederholte ich die Kapitel über die Niere und die Blase in meinem Lehrbuch der Pathophysiologie. Nach einem Monat konnte sie ihre Besuche in meiner Praxis wieder aufnehmen, wo ich sie jetzt zweimal pro Woche behandelte. Sie war deutlich gebessert. Sie erzählte, ihr Glas- gefäss sei schon voller kleiner Steine und sie würde bald ein zweites Glas brauchen. Als ich in die Sommerferien ging, war sie mehr oder weniger beschwerdefrei. Ihre zuvor dick angeschwollenen Beine hatten wieder die normale Form; sie hatte über $12 \mathrm{~kg}$ Gewicht verloren, was hauptsächlich aus angestautem Urin bestanden hatte; sie sah frisch aus und hatte rosige Wangen.

Eines Tages nahm ich sie mit zu meinem Urologen, der die Diagnose der Uniklinik bestätigt und mir erklärt hatte, dass hier keinerlei Behandlungsmöglichkeiten mehr bestünden. Er machte neue Röntgenbilder von ihren Nieren und war sehr erstaunt, dass jetzt beide Nierenbecken fast normal aussahen. Er fragte: «Was hast Du mit der Frau gemacht?»

"Akupunktur», sagte ich; aber er wollte mit nicht glauben.

Der Patientin ging es weiterhin gut. Sie kam anschliessend einmal monatlich zur Nachbehandlung. Gelegentlich hatte sie kleinere Rückfälle, die ich mit einigen Akupunkturen leicht beseitigen konnte. Jetzt, nach über 35 Jahren, ist sie bei bester Gesundheit und eine stolze Grossmutter von sechs Enkeln. Wie früher ist sie eine fromme Frau und davon überzeugt, dass ihre Gebete geholfen haben, mich und die richtige Behandlung $\mathrm{zu}$ finden. Augenzwinkernd versichert sie: «Ich bete immer fleissig für Sie mit, Herr Doktor. Es könnte ja sein, dass ich Sie noch mal brauchen werde!»

\section{Die Logik der Forschung}

In seinem Werk «Die Logik der Forschung» schreibt Karl Popper, dass er sich der Problematik der Induktion durchaus bewusst sei [24]. Er sagt frei heraus: "Ich lehne eine induktive Logik ab» [25]. Popper geht kurz auf den Positivismus ein, der von Descartes' res cogitans und res extensa, der Trennung zwischen Seele und Körper, stammt. Er schlägt eine mo- difizierte Version der Deduktion vor, bei der induktiv gewonnene Annahmen falsifizierbar sein müssen, was bedeutet, dass dabei der Satz vom Widerspruch ins Spiel kommt.

Der Atomphysiker Werner Heisenberg erklärte 1958 [26]:

Während die alten Griechen versucht hatten, Ordnung in der unendlichen Vielfalt der Dinge und Ereignisse zu finden und dabei nach einem grundlegenden einheitlichen Prinzip suchten, versuchte Descartes Ordnung durch eine fundamentale Trennung zu schaffen, die Polarität von «res cogitans» und «res extensa», und die $\mathrm{Na}$ turwissenschaft konzentrierte ihre Interessen auf die «res extensa». Der Einfluss der Cartesischen Trennung auf das menschliche Denken kann kaum überschätzt werden, aber es ist eben diese Trennung, die wir aus der Sicht der Entwicklung der modernen Physik kritisieren müssen. (...) Eine der späteren Folgerungen dieser Descartes'schen Ansicht war, dass wenn Tiere einfach als Maschinen zu betrachten waren (wie er annahm), schwierig war, dasselbe nicht auch vom Menschen zu denken. Weil, andererseits, die "res cogitans» und die «res extensa» ihrem Wesen nach als völlig verschieden angesehen wurden, schien es nicht möglich, dass beide gegenseitig aufeinander einwirken können. Um eine völlige Parallelität zwischen den Erfahrungen der Seele bzw. des Geistes aufrecht $\mathrm{zu}$ halten, mussten die Seele und der Geist (engl. mind) in ihren Handlungen völlig von Gesetzen bestimmt werden, die den Gesetzen der Physik und Chemie gehorchen. Hier stellte sich die Frage nach der Möglichkeit eines «freien Willen». Offensichtlich ist die ganze Beschreibung ziemlich künstlich und zeigt so die schweren Fehler der Descartes'schen Position.

\section{Heisenberg fährt fort [26]:}

Wir dürfen nicht übersehen, dass die Naturwissenschaft von Menschen gemacht ist. Die Naturwissenschaft beschreibt und erklärt Natur nicht einfach, sie ist ein Teil der Wechselwirkung zwischen der Natur und uns selbst, sie beschreibt Natur so, wie sie unserer Methode des Fragens entspricht. ... Die kartesische Spaltung ... ist während drei Jahrhunderten tief ins menschliche Bewusstsein gedrungen, und es wird lange dauern, bis sie durch eine wirklich andere Einstellung zum Problem der Wirklichkeit ersetzt werden kann.

Popper erklärt: «Meine Aufgabe, wie ich sie sehe, ist nicht die Abschaffung der Metaphysik» [27]. Und weiter: «Ausser konsistent zu sein, muss ein 
empirisches System eine weitere Bedingung erfüllen: es muss falsifizierbar sein ... Behauptungen, die nicht der Bedingung der Konsistenz genügen, verfehlen es, zwischen zwei Behauptungen innerhalb der Gesamtheit aller möglichen Behauptungen zu differenzieren» [28].

Wir werden Letzteres in Verbindung mit den Resultaten der GERACStudie zu diskutieren haben. Die Tatsache, dass die echte AkupunkturGruppe und die Placebo-Gruppe die Aufzeigung hinreichender Unterschiede verfehlt haben, zeigt, dass die bei der GERAC-Studie verwendeten Theorien nicht konsistent waren. Kurz gesagt: Die Überlegungen der Strategen der GERAC-Studie zu den Grundlagen der chinesischen Akupunktur waren falsch [29].

\section{Zur Theorie der chinesischen Medizin}

Womit befasst sich die chinesische Medizin? Ihre Grundregeln [30] lauten:

- Der menschliche Organismus ist ein einheitliches Ganzes.

- Die Beziehungen zwischen dem Menschen und der Natur sind einheitlich.

Aufgrund meiner eigenen Erfahrungen mit chinesischer Medizin in Europa und im Fernen Osten betone ich, dass die Descartes'sche Trennung zwischen res cogitans und res extensa, zwischen Geist und Materie, Seele und Körper, die die westliche Naturwissenschaft nach wie vor beherrscht, für die chinesische Heilkunde Zhong $Y i$ 中醫 (nicht etwa «TCM», denn die drei Buchstaben sind eine Verwechslung des Originalbegriffs!) weitgehend unbekannt ist.

Fahren wir hier fort, wo wir oben abgebrochen haben: Nachdem aus Shen 神 Himmel (Tian 天), Tugend bzw. Wahrheit (De 德), die Erde (Di 地), Yin-Yang 陰陽, $Q i$ 氣, Jing (Essenz) 精, Hun 魂, Po 魄, Xin 心, $Z h i$ 志 und $S i$ 思 entstanden sind, entwickeln sich aus Yin-Yang 陰陽 die Fünf Zang (五藏) (Speicherorgane) und die Sechs Fu (六腑) (Hohlorgane) sowie die Blutgefässe Xue Mai 血脈 (oder Jingmai 經脈): Taiyang 太陽, Shaoyang 少陽, Yangming 陽明, Taiyin 太陰, Shaoyin 少陰, Jueyin 厥陰.

Gemäss Kapitel 12, Jingmai 經脈, des Ling-Shu-Jing sind mit diesen Jingmai 經脈 die folgenden 11 inneren Organe verbunden: 膀胱 Pang Guang (Harnblase), 膽 Dan (Gallenblase), 胃 Wei (Magen), 脾 Pi (Milz), 腎 Shen (Niere), 肝 Gan (Leber), 小腸 Xiao Chang (Dünndarm), 大腸 $\mathrm{Da}$ Chang (Dickdarm), 肺 Fei (Lunge), 心 Xin (Herz), 心包 Xin Bao (Perikard). Die 三焦 San Jiao (Drei Erwärmer) bilden die Umhüllung der 11 inneren Organe.

Von hier und von den sechs klimatischen Störungen Liu Yin 六淫 (Wind 風 feng, Kälte 寒 han, Sommerhitze shu 暑, Feuchtigkeit 濕 shi, Trockenheit 燥 zao, Feuer 火 huo) entstehen in Verbindung mit Schleim 痰飲 tanyin, Blutgerinnseln 瘀血 yuxue, Infektionen, Insekten- und Tierbissen, mentaler Erschöpfung, falscher Ernährung, Verletzungen und Parasitenbefall Krankheiten, die ausnahmslos in die universelle Ordnung des Shen 神integriert werden können. Die Evidenz der chinesischen Medizin beginnt mit den Acht Leitprinzipien Ba Gang 八網, die entsprechend dem aristotelischen Satz vom Widerspruch in gegensätzlichen Paaren angeordnet sind:

- 表裡 Biao-Li (Oberfläche-Inneres)

- 寒熱 Han-Re (Kälte-Wärme)

- 虛實 Xu-Shi (Leere-Fülle)

- 陰陽 Yin-Yang (Yin-Yang)

Mittels Beurteilung des Zungenbildes und mit Pulsdiagnostik werden alle oben aufgeführten Phänomene, die Organe und Teile gemeinsam analysiert, um das zu differenzieren, was im chinesischen Sprachgebrauch Evidenz heisst, und was häufig genug der medizinischen Wahrheit entspricht. Durch Deduktion gewonnene Ableitungen unterliegen ausschliesslich dem aristotelischen Satz vom
Widerspruch, was sich hier bezüglich der chinesischen Heilkunde deutlich zeigt. Hier wird sozusagen eine Symptomdifferenzierung der diagnostischen Wahrheit vorgenommen, in die das Bian-Zheng 辩證 einmündet. Aus dem Bian-Zheng wird eine zugleich passende wie evidente Therapie mit Akupunktur, Moxibustion, Kräuterrezepten oder Tui-Na-Massage deduziert. Bei jedem einzelnen diagnostischen und therapeutischen Schritt bleiben Yin und Yang als

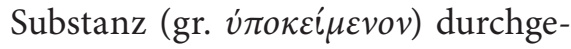
hend präsent und erkennbar. Allein auf dieser Basis ist die chinesische Medizin klinisch erfolgreich, und zwar deshalb, weil sie sich methodisch und logisch auf die Deduktion stützen kann [31].

Es sei erwähnt, dass das Schriftzeichen Zheng 證 in Bian-Zheng 辨證 «Evidenz» bzw. «Beweis» heisst. Die heute im Westen verbreitete Übersetzung von Bian-Zheng 辩證 mit «Muster» (engl. pattern) ist unzutreffend und zeigt ein Missverstehen der chinesischen Heilkunde an [32, 33]. Zheng 證 ist das erste Zeichen des modernen chinesischen Binoms Zhengju 證据 (證據), das mit «Evidenz», «Beweis» oder «Zeugnis» zu übersetzen ist [34]. Somit ist BianZheng 辨證 ein diagnostisches Verfahren, das auf Deduktion beruht und über den aristotelischen Satz vom Widerspruch mittels gegensätzlicher Krankheitszeichen logisch-analytisch Evidenz ermöglicht. Dies beweist, dass Bian-Zheng 辩證 wahrheitsgetreue Einsicht hinsichtlich der Diagnostik vermittelt, nämlich wirkliche Erkenntnis der vorliegenden spezifischen und individuellen Krankheitseinheit im Sinne der traditionellen chinesischen Heilkunde. Es stimmt überein mit den aristotelischen Anforderungen für einen verlässlichen Beweis, und es entspricht der Ansicht Karl Poppers hinsichtlich der gleichen wissenschaftlichen Aufgabe. Die logische Struktur solcher Evidenz ist grundlegend anders als die Strukturen moderner westlicher Diagnosen. 
Es handelt sich nämlich um einen anderen Typ diagnostischer Evidenz verglichen mit dem, was die EBM im Auge hat; denn es ist von der Natur als Ganzem deduziert, ohne dass es der kartesischen Trennung zwischen Geist und Materie, Seele und Körper zum Opfer fällt. Diese Evidenz geht nicht von isolierten Teilen des menschlichen Körpers aus, wie die meisten Spezialgebiete der modernen orthodoxen Heilkunde und wie die EBM, die auf mehr oder weniger vereinzelten Teilen basiert, auf Akzidenzien $(\sigma v \mu \beta \varepsilon \beta \eta \kappa o ́ \varsigma)$ im aristotelischen Verständnis, oft, ohne dass sich zwischen den Teilen noch logische Verbindungen erkennen lassen, was ich im Folgenden genauer erläutern werde.

\section{Evidence-Based Medicine und Medizinische Wissenschaft}

Die Definition der EBM [35] heisst: «Die EBM wurde definiert als die gewissenhafte, genaue und sorgfältige Anwendung der besten Evidenz bezüglich der Entscheidungsfindung hinsichtlich der Behandlung individueller Patienten.»

Dies scheint ein hervorragendes Ziel zu sein, das begeistert unterstützt werden muss. Das Zitat stammt aus «Essential Evidence-Based Medicine» von Dan Mayer, Professor der Emergency Medicine, Albany Medical College, Albany, NY, USA. Professor Mayer lobt die EBM als einen «Paradigmenwechsel, der das Ende des traditionellen hierarchischen Systems in der ärztlichen Praxis und die Annahme der wissenschaftlichen Methodik als beherrschendes Prinzip bei Fortschritten auf dem Gebiet der Medizin» darstelle. Das Problem ist indessen, dass die EBM, gemessen an aristotelischen Standards, weder gewissenhaft noch genau oder sorgfältig ist - ein Aspekt, der genauer zu untersuchen sein wird.

Die britische Professorin für Primary Health Care, Trisha Greenhalgh, vermisst in der oben zitierten
Definition von EBM «eine sehr wichtige Eigenschaft, nämlich die Verwendung der Mathematik». Sie schlägt eine andere Definition vor [36]: «Evidence-Based Medicine (EBM) ist die Anwendung der mathematischen Einschätzung von Nutzen und Schaden, gewonnen aus hochqualifizierter Forschung an Beispielen aus der Bevölkerung bezüglich der Entscheidungsfindung bei Diagnose, Erforschung oder Behandlung individueller Patienten.»

Die Frage ist: Von welcher Art und Weise ist eine solche wissenschaftliche Methode? Und womit befasst sich Wissenschaft der Medizin ganz allgemein? Beruht sie auf randomisierten Doppelblindstudien mit statistischen Dokumentationen einschliesslich der Sammlung einer Menge mehr oder weniger unproduktiver Daten? Oder könnte darin ein Verständnis des ganzen individuellen Patienten enthalten sein - wie in der chinesischen Heilkunde? Letzteres wäre wichtig, um den Patienten als Persönlichkeit zu verstehen, wobei seine Natur richtig erfasst und seine individuelle Krankheit erkannt würden.

Wenn wir anhand der Definition von Professor Greenhalgh fragen, ob in der EBM ein Verständnis des ganzen Menschen enthalten ist, müssen wir dies zu unserem Bedauern verneinen. Mathematik hat mit der Entwicklung des Menschen in der Natur nichts zu tun. Aristoteles kritisiert ausdrücklich die Verwendung von Mathematik in seiner Metaphysik (Buch I, Kapitel V, 3-10) [37], weil Zahlen keine Begründungsfunktion hinsichtlich der ersten Prinzipien haben [38]. Mein wichtigster Einwand ist, dass die EBM auf der Descartes'schen Trennung von Materie und Bewusstsein (res extensa und res cogitans) beruht und dementsprechend kein Ganzes sein oder verstehen, sondern im Sinne der aristotelischen Logik nur aus Teilen, d.h. aus Akzidenzien, arrangiert sein kann.

Unglücklicherweise wird der Begriff des Ganzen von modernen or- thodoxen Medizinern mit Skepsis betrachtet. Etliche westliche Ärzte argumentieren: «Das Ganze gibt es nicht, weil wir es nicht erkennen können.» Doch das klingt primitiv, indem es vorspiegelt, dass nur solche Sachen existieren können, die von Menschen erkannt werden; es enthält somit eine pseudowissenschaftliche Unterstellung. Wir werden sehen, dass wahre Wissenschaft nicht ohne den Begriff des Ganzen auskommt, eine Tatsache, die hartgesottenen Szientisten Kopfzerbrechen verursachen mag. Wir lernen von Aristoteles, dass die Natur (Physis $\varphi v i \sigma l \varsigma)$ notwendigerweise auf Metaphysik beruht. Seine Vorlesung über Natur, $\Phi \curlyvee \Sigma I K H \Sigma A K P O A \Sigma E \Omega \Sigma$, vermittelt die Regeln und Definition von Raum und Zeit als Anfangspunkt abendländischer Wissenschaft, gültig für alle Nachfolger einschliesslich Galileo [39]. Wir dürfen Newton und Einstein hinzufügen.

Der deutsche Philosoph Martin Heidegger hat den Begriff «Wissenschaft» folgendermassen erklärt [40]: «Wissenschaft ist das Ganze eines Begründungszusammenhangs, in welchem die Gegenstände der Wissenschaft im Hinblick auf ihren Grund vorgestellt und das heisst begriffen werden.»

Welches ist der Grund der klinischen Medizin? Beruht sie auf der Anwendung mathematischer Einschätzungen? Keineswegs. Sie basiert vielmehr auf dem individuellen Patienten als Ganzem. Wäre das neue Paradigma der EBM so geartet, könnte es als wichtiger wissenschaftlicher Durchbruch begrüsst werden. Es sieht aber so aus, als wäre die EBM vor allem mit Prozentzahlen befasst, die aus statistischen Berechnungen stammen. Der ganze individuelle Patient existiert nach Ansicht der EBM nicht, weil er (bzw. sie) als getrennte Dimensionen von Körper und Seele schon festgelegt und in zahlreiche Einzelteile, laut Aristoteles in Akzidenzien $(\sigma v \mu \beta \varepsilon \beta \eta \kappa o ́ \varsigma)$, die kein eigenes Sein haben, zerlegt worden ist. Diese Teile werden von der EBM irrtümlich als 


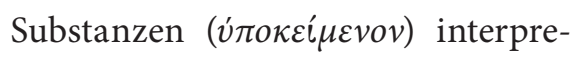
tiert, von wo aus die moderne medizinische «Beweisführung» beginnt. Wo ist der ganze individuelle Patient in der EBM geblieben? Nirgends! Er ist nach Meinung der EBM nicht etwa verschwunden, er hat nie existiert. Wir werden sehen, dass dies ein Trugschluss ist, ein logischer Fehler.

Der deutsche Atomphysiker und Philosoph Carl Friedrich von Weizsäcker schrieb 1971 in seinem Werk «Die Einheit der Natur» [41]:

Wenn ich es richtig sehe, erweist sich (...) der Vorwurf gegen eine verbreitete Tendenz in der Medizin, sie sehe die Person oder den Geist nicht, letztlich nicht als identisch mit dem Vorwurf, sie sei Naturwissenschaft, sondern allenfalls identisch mit dem Vorwurf, sie sei noch nicht naturwissenschaftlich genug, nämlich noch nicht hinreichend gute Naturwissenschaft, das heisst, nicht genügend selbstkritisch.

Hat die EBM diese Stufe von Selbstkritik schon erreicht oder muss die EBM vielleicht modifiziert und verbessert werden, um den geforderten Qualitätsgrad zu erlangen?

Professor Dan Mayer nennt in seinem Buch drei Fertigkeiten, über die der ärztliche Praktiker verfügen soll:

- Beherrschung der Information (BI),

- kritische Bewertung (KB) und

- Übertragung von Wissen (ÜW).

Das Erste ist die Fähigkeit, die medizinische Fachliteratur zu durchsuchen; das Zweite ist die Entwicklung kritischen Denkens hinsichtlich des Inhalts der medizinischen Literatur; das Dritte ist die Anwendung der gefundenen und kritisch bewerteten Information auf den Patienten [35]. Das mache ich in diesem Artikel, indem ich den ganzen individuellen Patienten berücksichtige.

Ich möchte eine vierte Fähigkeit zu Professor Mayers Liste fügen, nämlich:

Jeder EBM-Praktiker sollte sich mit chinesischer Medizin vertraut machen und mit dem, was dort Bian-Zheng 辨證 genannt wird, um zunächst über eine verlässliche

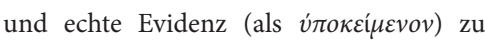
verfügen, die später unschwer mit den einzelnen Errungenschaften der modernen westlichen Medizin (als $\sigma v \mu \beta \varepsilon \beta \eta \kappa o ́ \varsigma$ ) verbunden werden kann, wobei sichergestell werden muss, dass chinesische Heilkunde und Bian-Zheng vollkommen verstanden worden sind und auch das Erste Prinzip

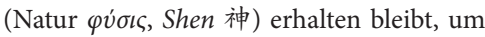
den individuellen Patienten als Ganzes zu verstehen, ohne der kartesischen Trennung zu erliegen [31, 42].

Übrigens erwähnt Professor Mayer in seinem Buch beiläufig die chinesische Medizin, aber er wiederholt falsche Annahmen über Energiefluss und Energiekanäle, wie es westliche Akupunkteure tun (was die Ursache für die Verwirrung der deutschen GERAC-Studie war). Darüber hinaus ist er mit den Anfängen der chinesischen Anatomie im Jahre 16 n.Chr. nicht vertraut, worüber ich in dieser Zeitschrift schon publiziert habe [43].

Im Jahr 1931 schrieb der renommierte deutsche Philosoph Karl Jaspers, selbst Arzt und Psychiater [44]:

Eine Verkehrung des Sinnes von Wissenschaft geht heute durch die Welt. Wissenschaft geniesst einmal einen ausserordentlichen Respekt. Da Massenordnung nur durch Technik, Technik nur durch Wissenschaft möglich ist, herrscht im Zeitalter ein Glaube an Wissenschaft. Da aber Wissenschaft allein zugänglich ist durch methodische Bildung, das Staunen vor ihren Resultaten noch kein Teilnehmen an ihrem Sinn ist, so ist dieser Glaube Aberglaube. Eigentliche Wissenschaft ist das Wissen mit dem Wissen der Weisen und Grenzen des Wissens. Wird aber an ihre Resultate geglaubt, die nur als solche, nicht in der Methode ihres Erwerbs gekannt werden, so wird in imaginärem Missverstehen dieser Aberglaube zum Surrogat echten Glaubens. Die Macht dieses Aberglaubens befällt alle Menschen, auch die Gelehrten ...

Befürworter der EBM sollten sich diese Äusserung vor Augen halten, wenn sie über medizinische Wissenschaft reden. Jaspers bezieht sich in seinem Buch «Die Geistige Situation der Zeit», aus dem das Zitat stammt, offensichtlich auf das Erbe des Aristoteles, dessen Vorlesung über Natur $(\Phi \Upsilon \Sigma I K H \Sigma A K P O A \Sigma E \Omega \Sigma$ ) Parallelen $\mathrm{zu}$ frühen chinesischen Medizintex- ten wie dem Huangdi Neijing Lingshu (Lingshujing) aufweist, was im Voranstehenden ausgeführt worden ist.

\section{Literatur}

1 Die Fragmente der Vorsokratiker, Griechisch/ Deutsch. Band I, Herakleitos (Heraklit) 123. Zürich, Weidmann, 1996, p 178.

2 Heisenberg W: Physics and Philosophy. London, Penguin Books, 1958/1989, p 129.

320 Minuten. Zürich, 18. Mai 2009, p 8.

$\checkmark 4$ Schnorrenberger CC: Zu den Quellen der Chinesischen Medizin. Falsche Übersetzungen chinesischer Schriftzeichen beeinträchtigen die Aussagekraft der deutschen Gerac-Studie («Modellvorhaben»). Schweiz Zschr Ganzheitsmedizin 2005;17:150-156.

5 Lossau N: Die meisten Akupunkteure sind schlecht ausgebildet. Hamburg, Die Welt, 30.01.2002.

6 Haake M, Müller HH, Schade-Brittinger C, Basler HD, Schäfer H, Maier C, Endres HG, Trampisch HJ, Molsberger A: German Acupuncture Trials (GERAC) for chronic low back pain: randomized, multicenter, blinded, parallel-group trial with 3 groups. Arch Intern Med 2007;167:1892-1898.

7 Ernst E: Medicine Man: An end to 'free' acupuncture sessions? No wonder doctors and patients got the needle. 16.03.2004. www.guardian.co.uk.

8 Birch S: Reflections on the German Acupuncture Studies. J Chin Med 2007;83:12-17.

9 Huangdi Neijing Lingshu (Neijing Lingshu Minghui Jiyin 內經霝樞名彙集引), Nachdruck. Taichung/Taiwan, 1980, Teil 1: Originaler Text, p 1.

10 Wieger L: Chinese Characters. Their Origin, Etymology, History Classsification and Signification. New York, Dover Publications, Inc., 1965, p 29.

11 Huangdi Neijing Lingshu (Neijing Lingshu Minghui Jiyin 內經霝樞名彙集引), Nachdruck. Taichung/Taiwan, 1980, Teil 1: Originaler Text, p 31.

12 Mathews' Chinese-English Dictionary, ed 12. Cambridge, MA, Harvard University Press, 1972, p 882.

13 Lao Zi Zheng Wen 老子徵文, Gesammelte Texte des Lao Zi, kommentiert von Meng Wentong 蒙文通. Taipei, 1998, p 110.

14 Aristotle: On Sophistical Refutations ( $О Ф I \Sigma T I K \Omega N$ E $\Lambda E \Gamma X \Omega N)$. Book V, 166b 8. Cambridge, MA, Harvard University Press, 1955, pp 24-26.

15 Aristotle: Physics (Lecture on Nature $\Phi \Upsilon \Sigma I K H \Sigma A K P O A \Sigma E \Omega \Sigma$ ), Book I, chapter II: 185a, 30; chapter III: 186a, 34; 186b, 18-20. Cambridge, MA, Harvard University Press, 1929, pp 20, 32, 34.

16 Aristotle: Metaphysics (TSN META TA ФऽIKA), Book VI, chapter II, 1026a, 4-13; chapter II, 1026b, 4. Cambridge, MA, Harvard University Press, 1933, pp 299-305.

17 Die Fragmente der Vorsokratiker, Griechisch/ Deutsch. Band I. Zürich, Weidmann, 1996. Band I, pp 213, 455, 478; Band II, pp 69, 182. 
18 Aristotle: Posterior Analytics (ANA YYTIK $\Omega N$ $\Upsilon \Sigma T E P \Omega N)$. Cambridge, MA, Harvard University Press, 1960, vol II, 75b14f., pp 62-63.

19 Aristotle: Physics (Lecture on Nature $\Phi \Upsilon \Sigma I K H \Sigma$ AKPOA $\Sigma E \Omega \Sigma$ ), Book V, chapter 4, 227b; Book VII, chapter 3, 247b. Cambridge, MA, Harvard University Press, 1929.

20 Aristotle: Topics (TOПIK $\Omega N$ ). Vol. II, 162a 17. Cambridge, MA, Harvard University Press pp 724-725.

21 Popper KR: The Logic of Scientific Discovery. London, Hutchinson, 1980, p 280

22 Aristotle: Physics (Lecture on Nature $\Phi \Upsilon \Sigma I K H \Sigma$ $A K P O A \Sigma E \Omega \Sigma$ ), Book I, chapter 1, 184a 10-27; pp 10-13. Zitierte englische Übersetzung von Wickstedt PH, Cornford FM.

23 Aristotle: Physics (Lecture on Nature $\Phi \Upsilon \Sigma I K H \Sigma$ $A K P O A \Sigma E \Omega \Sigma$ ). 184a, 25; 186b, 25; 195a, 21; 207a 9. Cambridge, MA, Harvard University Press, 1929, pp 10, 34, 132, 252.

24 Popper KR: The Logic of Scientific Discovery. London, Hutchinson, 1980, pp 27-29.

25 Popper KR: The Logic of Scientific Discovery. London, Hutchinson, 1980, p 35.

26 Heisenberg W: Physics and Philosophy. London, Penguin Books, 1958/1989, pp 40-42.
27 Popper KR: The Logic of Scientific Discovery. London, Hutchinson, 1980, p 37.

28 Popper KR: The Logic of Scientific Discovery. London, Hutchinson, 1980, p 92.

29 Lehmann H: Am Anfang war ein Scharlatan. Dtsch Ärztebl 2010;107:A1454-1457.

30 Schnorrenberger CC: Lehrbuch der chinesischen Medizin für westliche Ärzte. Stuttgart, Hippokrates, 1979, p 29.

31 Schnorrenberger CC: Syndrome acupuncture (Bian-Zheng Zhen-Jiu) and herbal prescription (Bian-Zheng Fang-Ji) - a new medical paradigm for the West. Schweiz Zschr Ganzheitsmedizin 2006;18:153-158.

32 Kapchuk T: Chinese Medicine. The Web that Has no Weaver. London, Rider, 1983, pp 178241.

33 Maciocia G: The Foundations of Chinese Medicine. Edinburgh, Churchill-Livingstone, 1989, $\mathrm{p} 1$.

34 Mathews' Chinese-English Dictionary. Cambridge, MA, Harvard University Press, 1972, p 45.

35 Mayer D: Essentials of Evidence Based Medicine, ed 2. Cambridge, Cambridge University Press, 2010, p 10.
36 Greenhalgh T: How to Read a Paper. The Basics of Evidence-Based Medicine, ed 4. Chichester, Wiley-Blackwell BMJ Books, 2010, p 1.

37 Aristotle: Metaphysics (TSN META TA Фऽ $\Sigma I K A)$, Book I, chapter V, 986a, 5. Cambridge, MA, Harvard University Press, 1933, p 32.

38 Aristotle: Metaphysics (TSN META TA $\Phi \Upsilon \Sigma I K A)$, Book XIV, chapter V, 1092b, 11-13. Cambridge, MA, Harvard University Press, 1933, p 294

39 Heidegger M: Der Satz vom Grund. Pfullingen, Neske, 1957, p 111.

40 Heidegger M: Identität und Differenz. Pfullingen, Neske, 1965, p 50.

41 von Weizsäcker CF: Die Einheit der Natur. München, Hanser, 1971, p 291.

42 Schnorrenberger CC: Chen-Chiu. The Original Acupuncture. A New Healing Paradigm. Boston, MA, Wisdom Publications, 2004.

43 Schnorrenberger CC: Zur Entwicklung der chinesischen Medizin im Westen, Teil 1 und Teil 2. Schweiz Z Ganzheitsmed 2010;22:157165 und 217-221.

44 Jaspers K: Die geistige Situation der Zeit. Berlin, De Gruyter, 1931, p 138 